\title{
Acantoma de células claras abdominal. Lesión infrecuente en localización atípica
}

\author{
Francisco Javier Torres-Gómez 1*, Pilar Fernández-Machín ${ }^{1}$, Carolina Cantalejo-Rodríguez ${ }^{2}$
}

Resumen: Introducción. El acantoma de células claras es una lesión tumoral benigna relativamente infrecuente que muestra unas características histológicas que permiten su diagnóstico. Método. Presentamos el caso de un acantoma de células claras abdominal, localización infrecuente, en un paciente de 72 años. Resultado. El diagnóstico fue establecido tras el pertinente estudio histológico pues la clínica inespecífica y la localización atípica impedían la realización de un correcto juicio clínico. Conclusiones. Pretendemos con la presentación del presente caso poner de manifiesto la histología tan peculiar de esta lesión al tiempo que, a través de una amplia revisión bibliográfica, mostramos distintas localizaciones y presentaciones atípicas de dicha entidad.

Palabras clave: acantoma; células claras; abdominal; glucógeno

Abstract: Introduction. Clear cell acanthoma is a relatively rare benign tumoral lesion showing histological features that allow diagnosis. Method. We report the case of abdominal clear cell acanthoma, unusual location in a patient of 72 years. Results. The diagnosis was established after the relevant histological study as nonspecific clinical and atypical location prevented the realization of a proper clinical trial. Conclusions. We intend with the presentation of this case highlight the peculiar histology of this lesion while, through an extensive literature review, we show different locations and atypical presentations of this entity.

Keywords: acanthoma; clear cells; abdominal; glucogen

Fecha de envío: 3 de abril de 2016- Fecha de aceptación: 31 de mayo de 2016

\section{Introducción}

El acantoma de células claras es una infrecuente lesión tumoral benigna cuya etiología neoplásica o traumática-reactiva es aún debatida. Se trata de una lesión epidérmica de diagnóstico histológico pues sus rasgos clínicos impiden su certero diagnóstico. Se suele asociar a una típica localización en piernas de mujeres añosas. Localizaciones distintas dificultan más su diagnóstico para el que, a pesar de su típica histología, es necesario realizar diagnósticos diferenciales. Presentamos un caso de acantoma de células claras en la más que infrecuente localización en pared abdominal en un paciente varón y añoso. Aprovechamos la ocasión para hacer una revisión de la literatura y profundizar en el conocimiento de esta peculiar entidad.

\section{Método:}

Nuestro paciente es un varón de 72 años que consultó en el Servicio de Dermatología debido a una lesión popular pruriginosa de $0,4 \mathrm{~cm}$ de dimensión máxima con crecimiento progresivo a nivel de pared abdominal (Figura 1). En superficie se observaba una delgada capa queratósica. Con los juicios de queratosis seborreica o granuloma piógeno pero sin poder descartar que se tratara de un carcinoma de células basales, se decidió realizar la exéresis lesional y remitirla al laboratorio de Anatomía Patológica para realizar el estudio histológico correspondiente.

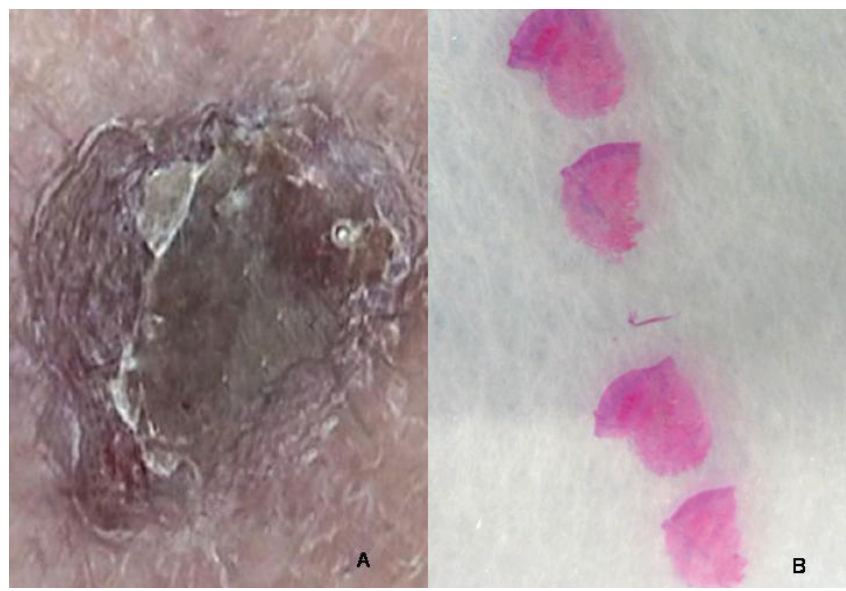

Figura 1: Acantoma de células claras abdominal. Imagen macroscópica (A) y macro-microscópica (B).

(1) Área de gestión Clínica de Biotecnología. Agencia Sanitaria Bajo Guadalquivir. Sevilla. Spain

(2) Servicio de Dermatología. Agencia Sanitaria Bajo Guadalquivir. Sevilla. Spain

*Autor de correspondencia: javiertorresgomez@yahoo.es 


\section{Resultado:}

El estudio microscópico demostró una hiperplasia epitelial irregular sin atipia, con discreta-moderada espongiosis y una llamativa exocitosis de leucocitos polimorfonucleares neutrófilos. En superficie se apreciaba una capa de hiperparaqueratosis con formación de microabscesos neutrofílicos focales. En el seno del epitelio se distinguían sábanas de queratinocitos con células claras, bien elimitados de los queratinocitos adyacentes y de aquellos del estrato basal epidérmicos que les servían de marco (Figuras 2-4). Tales hallazgos determinaron el diagnóstico de acantoma de células claras, corroborado por los resultados de la tinción de PAS llevada a cabo, mostrando la tinción rosada-magenta de los citoplasmas de las "células claras". En dermis, si bien se observaba una proliferación de pequeños vasos sin atipia, ésta no era muy evidente.

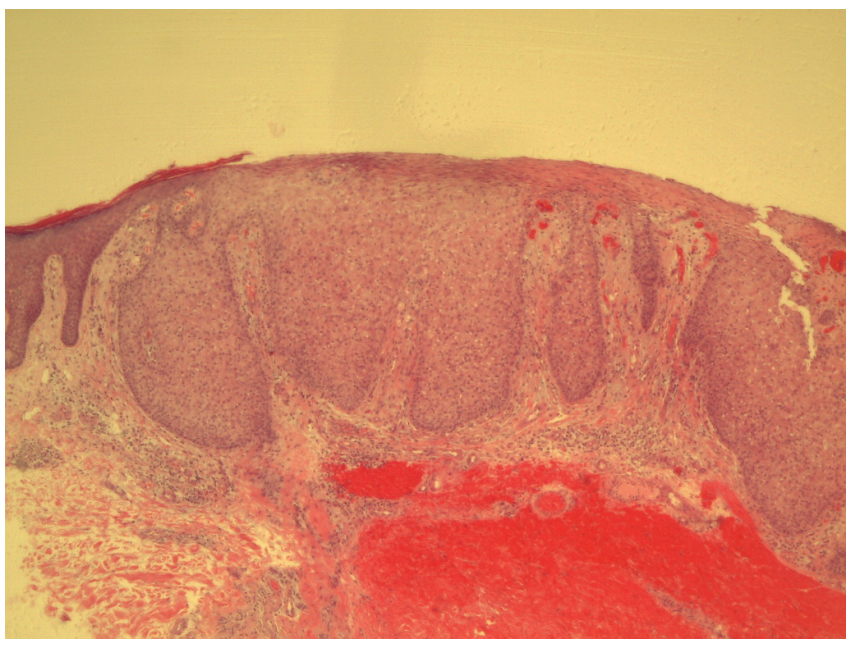

Figura 2: Acantoma de células claras abdominal. Silueta lesional en la que se observa una hiperplasia irregular de la epidermis con queratinocitos con citoplasma claro bien delimitados de los adyacentes. HE 40x.

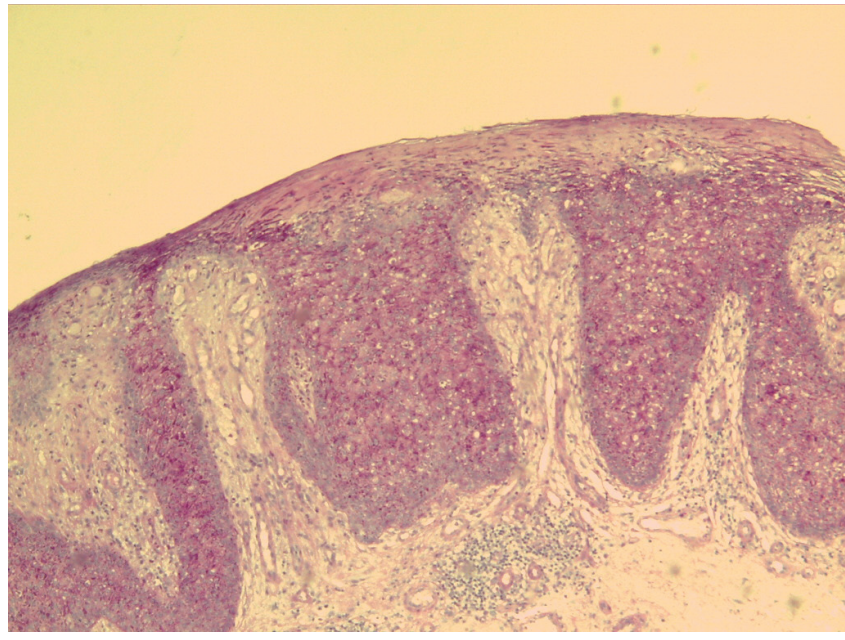

Figura 3: Acantoma de células claras abdominal. Con la tinción de PAS, las células claras adquieren una tonalidad citoplasmática rosada-magenta. PAS 40x.

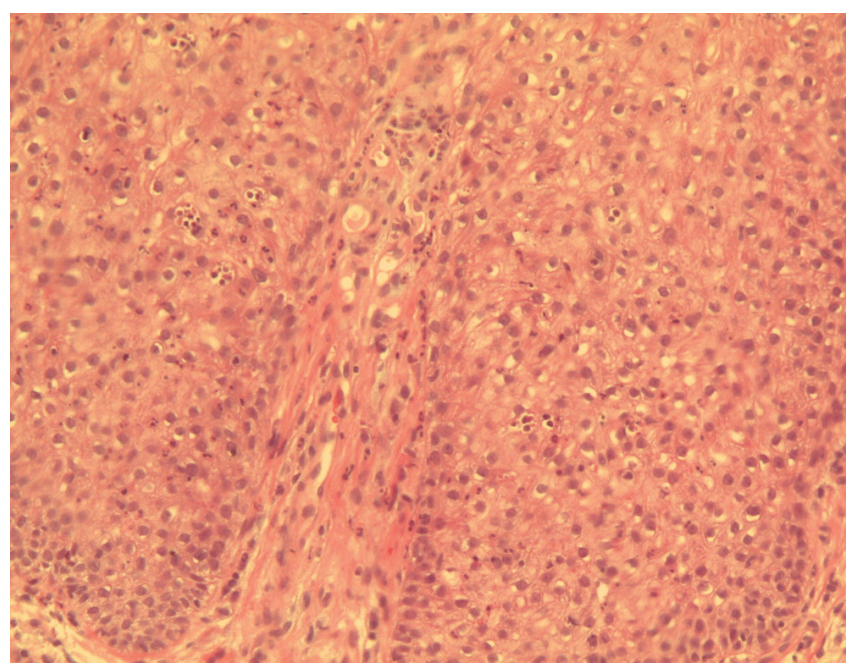

Figura 4: Acantoma de células claras abdominal. En el seno de la población de células claras se observan leucocitos polimorfonucleares neutrófilos aislados o formando pequeños grupos. HE 200x.

\section{Discusión y conclusiones:}

El acantoma de células claras, también denominado acantoma de células pálidas o acantoma de Degos en honor al autor que primero lo individualizó y describió en la literatura (Degos et al., 1962) es, como su nombre indica, una lesión hiperplásica o proliferativa epitelial (epidérmica) caracterizada por la presencia de células con citoplasma claro bien diferenciadas de los queratinocitos adyacentes (Degos et al., 1962; Fine \& Chernosky, 1969). Esta sencilla definición debe ser completada para individualizar una entidad para la que aun se discute su naturaleza neoplásica (benigna) o reactiva, relacionándola con la psoriasis. Por ello, es necesario precisar que las células claras se encuentran perfectamente delimitadas de los queratinocitos adyacentes y del estrato basal, que conserva características tintoriales intactas con hematoxilina-eosina (HE). La palidez citoplasmática característica es debida al acúmulo de glucógeno de los mismos, fácilmente demostrado con técnicas de PAS, que condicionan una coloración citoplasmática rosada-magenta (McKee et al., 2005; Kazakov et al., 2012; Lever et al., 2015). Pero es preciso precisar más aun en cuanto a la histología lesional pues distintos tipos de poroma podrían cumplir con los requisitos hasta ahora expuestos (Lever et al., 2015). Es muy frecuente, e incluso podría decirse que característica, la presencia de infiltración de la epidermis por leucocitos polimorfonucleares neutrófilos con o sin leucocitoclasia asociada, aislados o formando microabscesos (también frecuentes a nivel del estrato córneo hiperqueratósico-paraqueratósico), uno de los rasgos que, unido a la propia hiperplasia epitelial psoriasiforme y a la frecuente paraqueratosis superficial, permite postular relaciones etiológicas con la psoriasis (McKee et al., 2005; Kazakov et al., 2012; Lever et al., 2015). 
Cabe preguntarse si existen rasgos clínicos distintivos del acantoma de células claras. Independientemente del gran número de presentaciones y localizaciones descritas en la literatura, que posteriormente analizaremos, se trata de lesiones por lo general numulares y cupuliformes de pequeño tamaño $(2-4 \mathrm{~mm})$ que muestran una tonalidad rosada o rojizo pardusca, con trazas queratósicas o telangiectásicas superficiales frecuentes. Se trata, pues, de rasgos inespecíficos que impiden el diagnóstico clínico inicial, siendo necesario recurrir a la histología para realizar la caracterización lesional. Diagnósticos clínicos diferenciales realizados con frecuencia son queratosis seborreica, nevus epidérmico, eczema numular, la propia psoriasis y, como en nuestro caso, el granuloma piogénico.

El estudio histológico de distintos acantomas de células claras permite realizar correlaciones clínico-patológicas, la primera de ellas dirigida a justificar la tonalidad rosada o rojiza de gran número de especímenes. La misma puede estar relacionada con una evidente neovascularización que, en localización dérmica está presente en la mayoría de los casos. Se trata de vasos de pequeño calibre sin atipia endotelial, rodeados de una respuesta fibrótica de la lámina propia (Rotaru et al., 2014). Dicha respuesta tisular, además de la posible presencia de edema asociado, es propia de la presentación más frecuente del acantoma de células claras: en la pierna de mujer de edad avanzada. En tal caso, dichos cambios podrían ser del mismo modo atribuidos a problemas circulatorios (insuficiencia venosa) con los que podrían confundirse. El caso que presentamos, de localización abdominal, adolece de cambios tan marcados, reforzando la teoría previamente expuesta.

El diagnóstico de acantoma de células claras puede establecerse sin la concurrencia de técnicas histoquímicas y/o inmunohistoquímicas si bien con técnicas de PAS puede ponerse de manifiesto la presencia de glucógeno en el citoplasma de las células claras, confirmándose el diagnóstico (Desmons et al., 1977; McKee et al., 2005; Kazakov et al., 2012; Tempark \& Shwayder, 2012; Rotaru et al., 2014; Lever et al., 2015). La histoquímica también ha contribuido a aclarar la verdadera patogénesis lesional al demostrar la ausencia de fosforilasa en los queratinocitos "claros", poniendo de manifiesto la incapacidad de dichas células de degradar correctamente el glucógeno, fenómeno fisiológico tanto en los queratinocitos adyacentes como en aquellos que conforman el estrato basal que enmarca a las células claras.

La microscopía electrónica podría demostrar la presencia de vacuolas de glucógeno en las células claras pero no es necesario recurrir a un estudio tan detallado para alcanzar u diagnóstico correcto (Lever et al., 2015).
Como hemos comentado, no es necesario el concurso de técnicas inmunohistoquímicas con fines diagnósticos si bien en la literatura el acantoma de células claras ha sido intensamente estudiado desde esta perspectiva, caracterizando a la lesión de un modo más que minucioso (Desmons et al., 1977; Tempark \& Shwayder, 2012; Rotaru et al., 2014).

Son muchas y variadas las aproximaciones al acantoma de células claras desde el punto de vista dermatoscópico (Blum et al., 2001; Bugatti et al., 2003; Lacarrubba et al., 2003; Zalaudek et al., 2003; Miyake et al., 2014; Lyons et al., 2015; Tiodorovic-Zivkovic et al., 2015) siendo el patrón "en cuentas de collar" el más frecuentemente descrito. Algunos trabajos incluso plantean desde este punto de vista el diagnóstico diferencial con la psoriasis, entidad con la que se ha relacionado el acantoma de células claras (Blum et al., 2001; Bugatti et al., 2003; Zalaudek et al., 2003).

Existen numerosas variantes clínicas y asociaciones descritas del acantoma de células claras. Precisamente se ha descrito el desarrollo de un acantoma de células claras sobre una placa de psoriasis (Finch \& Tan, 2000). También se ha descrito a la asociación con Enfermedad de Bowen multifocal (Shirai et al., 2014). Generalmente hemos precisado que se trata de una lesión única de crecimiento lento y progresivo con localización preferente en la pierna de una mujer con edad media-avanzada. No obstante, se han descrito localizaciones tan heterogéneas como la axila (Lever et al., 2015), primer dedo del pie (Wang \& Chi, 2006), pezón (Nazzaro, 2014), el dorso de la mano (Cetinozman et al., 2016) e incluso en un injerto cutáneo (Wang \& Chi, 2005), siendo nuestro caso el primero descrito en pared abdominal. Llama la atención la descripción de una veintena de casos de acantomas de células claras múltiples haciendo hincapié algunas de ellas la rápida progresión presentada por algunas de las lesiones (Monari et al., 2010; Rotaru et al., 2014; Martinez Escaname et al., 2015).También has sido descritas variantes "gigantes" del acantoma de células claras asociadas a tratamiento con infliximab en un paciente con psoriasis, sin transcendencia pronóstica (Cavicchini et al., 2015) e incluso se han descrito lesiones con la misma histología inducidas por un dermatofibroma en crecimiento (Silverstein \& Marghoob, 2012).

Son muchas las variantes histológicas descritas, Ilamando especialmente la atención aquellas que mostraban pigmentación clínica, correspondiéndose aquella con la presencia de melanocitos dendríticos en distintos estratos epidérmicos, cuadro histológico muy cercano al descrito en el melanoacantoma (Pierard, 1991; Saeki et al., 2014; Jacyk et al., 2016). En otros casos, la pigmentación se debía al depósito de hemosiderina en torno a vasos de la dermis superficial y conformando una banda a nivel de dermis reticular en una localización expuesta a traumas como es el muslo (Bugatti 
\& Filosa, 2011). Por último, queremos destacar la asociación del acantoma de células claras con un siringofibroadenoma ecrino (Shalin et al., 2013).

No se han descrito casos de malignización del acantoma de células claras debiendo replantearse el diagnóstico en caso de ser demostrada agresividad o recidiva lesional no asociada a técnica de exéresis insuficiente. Si bien a priori no se contempla en las distintas clasificaciones el acantoma de células claras maligno, Lin y cols. (Lin et al., 2016) describen una lesión que podría ser encuadrada en esta definición.

Desde el punto de vista terapéutico son muchas y variadas las aproximaciones realizadas. Parece lógico pensar que la exéresis quirúrgica es el tratamiento de elección de una lesión hasta cierto punto inespecífica desde el punto de vista clínico que puede ser confundida con un carcinoma. En nuestro caso, como en casi todos se optó por esta opción, sin que se hayan descrito recidivas posteriores. Entre las alternativas con éxito recogidas para el tratamiento del acantoma de células claras destacamos la crioterapia (Altman et al., 1989; Betti et al., 1995), el fluoruracilo (Garcia Almagro et al., 1979), el láser de dióxido de carbono (Chi et al., 2005) o el calcipotriol tópico (Scanni \& Pellacani, 2014).

\section{Contribuciones}

El presente trabajo ha sido diseñado y escrito con el consentimiento y la participación de los tres autores sin que existan fuentes de financiación o posibles conflictos de interés que dificulten su publicación.

\section{Referencias}

Altman AR, Basler E \& Rosen T. (1989). Cryosurgical treatment of clear cell acanthoma. International journal of dermatology 28, 334-335.

Betti R, Bruscagin C, Inselvini E, Palvarini M \& Crosti C. (1995). SuCcessful cryotherapic treatment and overview of multiple clear cell acanthomas. Dermatologic surgery: official publication for American Society for Dermatologic Surgery [et al] 21, 342-344.

Blum A, Metzler G, Bauer J, Rassner G \& Garbe C. (2001). The dermatoscopic pattern of clear-cell acanthoma resembles psoriasis vulgaris. Dermatology 203, 50-52.

Bugatti L \& Filosa G. (2011). Hemosiderotic clear-cell acanthoma: a pigmented mimicker. Indian journal of dermatology 56, 426-427.

Bugatti L, Filosa G, Broganelli P \& Tomasini C. (2003). Psoriasis-like dermoscopic pattern of clear cell acanthoma. Journal of the European Academy of Dermatology and Venereology: JEADV 17, 452-455.
Cavicchini S, Nazzaro G \& Marchetti S. (2015). Fast-growing 'giant' clear cell acanthoma detected by dermoscopy during treatment with infliximab in a psoriatic patient. Journal of the European Academy of Dermatology and Venereology : JEADV 29, 1642-1644.

Cetinozman F, Jansen P \& Willemze R. (2016). Clear cell acanthoma on the dorsum of the hand. Journal of the European Academy of Dermatology and Venereology: JEADV 30, 378-380.

Chi CC, Wang SH \& Huang HS. (2005). Clear cell acanthoma successfully treated with a carbon dioxide laser. Dermatologic surgery : official publication for American Society for Dermatologic Surgery [et al] 31, 1355-1358.

Degos R, Delort J, Civatte J \& Poiares-Baptista A. (1962). Tumeur epidermique d'aspect particulier: acanthome á cellulles claires. Ann Dermatol Syphiligr 89, 361-371.

Desmons F, Breuillard F, Thomas P, Leonardelli J \& Hildebrand HF. (1977). Multiple clear-cell acanthoma (Degos): histochemical and ultrastructural study of two cases. International journal of dermatology 16, 203-213.

Finch TM \& Tan CY. (2000). Clear cell acanthoma developing on a psoriatic plaque: further evidence of an inflammatory aetiology? The British journal of dermatology 142, 842-844.

Fine RM \& Chernosky ME. (1969). Clinical recognition of clear-cell acanthoma (Degos'). Archives of dermatology 100, 559-563.

Garcia Almagro D, Bueno C, Corripio F, Lecona M \& Jaqueti G. (1979). [Multiple clear cell acanthoma in a patient with psoriasis. Treatment with 5-fluorouracil]. Medicina cutanea ibero-latino-americana $\mathbf{7}, 109-113$.

Jacyk WK, Baran W \& Essop A. (2016). Multiple pigmented clear cell acanthoma in an African patient. Journal of the European Academy of Dermatology and Venereology: JEADV 30, 494-496.

Kazakov DV, Spagnolo DV, Kacerovska D, Rychly B \& Michal M. (2012). Cutaneous type adnexal tumors outside the skin. Wolters Kluwer, Philadelphia.

Lacarrubba F, de Pasquale R \& Micali G. (2003). Videodermatoscopy improves the clinical diagnostic accuracy of multiple clear cell acanthoma. European journal of dermatology : EJD 13, 596-598.

Lever WF, Schaumburg-Lever G \& Gottlieb B. (2015). Histopathology of the Skin. 11th Edition. Philadelphia. 
Lin CY, Lee LY \& Kuo TT. (2016). Malignant Clear Cell Acanthoma: Report of a Rare Case of Clear Cell Acanthoma-Like Tumor With Malignant Features. The American Journal of dermatopathology 38, 553-556.

Lyons G, Chamberlain AJ \& Kelly JW. (2015). Dermoscopic features of clear cell acanthoma: five new cases and a review of existing published cases. The Australasian journal of dermatology 56, 206-211.

Martinez Escaname M, Arzberger E, Hofmann-Wellenhof R \& Massone C. (2015). Multiple clear-cell acanthomas. The Australasian journal of dermatology 56, e46-48.

McKee PH, Calonje E \& Granter SR. (2005). Pathology of the Skin: With Clinical Correlations. Elsevier Mosby, Philadelphia.

Miyake T, Minagawa A, Koga H, Fukuzawa M \& Okuyama R. (2014). Histopathological correlation to the dermoscopic feature of "string of pearls" in clear cell acanthoma. European journal of dermatology : EJD 24, 498-499.

Monari P, Farisoglio C, Gualdi G, Botali G, Ungari M \& Calzavara-Pinton P. (2010). Multiple eruptive clear cell acanthoma. Journal of dermatological case reports 4, 25-27.

Nazzaro G. (2014). Clear cell acanthoma of the nipple: another report from Italy. Anais brasileiros de dermatologia 89, 189.

Pierard GE. (1991). Melanocytes in clear cell acanthoma. The American Journal of dermatopathology 13, 430.

Rotaru M, lancu GM, Gheuca Solovastru L, Glaja RF, Grosu F, Bold A $\&$ Costache A. (2014). A rare case of multiple clear cell acanthoma with a relatively rapid development of the lower legs. Romanian journal of morphology and embryology = Revue roumaine de morphologie et embryologie 55, 1171-1179.
Saeki H, Matsuzaki H, Ito K, Nobeyama Y \& Nakagawa H. (2014). Pigmented clear cell acanthoma of the finger simulating pigmented nevus. International journal of dermatology 53, e579-581.

Scanni G \& Pellacani G. (2014). Topical calcipotriol as a new therapeutic option for the treatment of clear cell acanthoma. Anais brasileiros de dermatologia $\mathbf{8 9}, \mathbf{8 0 3 - 8 0 5 . ~}$

Shalin SC, Rinaldi C \& Horn TD. (2013). Clear cell acanthoma with changes of eccrine syringofibroadenoma: reactive change or clue to etiology? Journal of cutaneous pathology 40, 1021-1026.

Shirai A, Saeki H, Matsuzaki H, Ito K \& Nakagawa H. (2014). Multiple clear cell acanthoma associated with multiple Bowen's disease. International journal of dermatology 53, e386-388.

Silverstein D \& Marghoob A. (2012). Clear cell acanthoma induced by a dermatofibroma. Dermatology online journal 18, 14.

Tempark T \& Shwayder T. (2012). Clear cell acanthoma. Clinical and experimental dermatology 37, 831-837.

Tiodorovic-Zivkovic D, Lallas A, Longo C, Moscarella E, Zalaudek I \& Argenziano G. (2015). Dermoscopy of clear cell acanthoma. Journal of the American Academy of Dermatology 72, 547-49.

Wang SH \& Chi CC. (2005). Clear cell acanthoma occurring in a split-thickness skin graft. Plast Reconstr Surg 116, 146e-149e. Wang SH \& Chi CC. (2006). Clear cell acanthoma occurring on the hallux: the first case report. Journal of the European Academy of Dermatology and Venereology: JEADV 20, 1144-1146.

Zalaudek I, Hofmann-Wellenhof R \& Argenziano G. (2003). Dermoscopy of clear-cell acanthoma differs from dermoscopy of psoriasis. Dermatology 207, 428; author reply 429. 